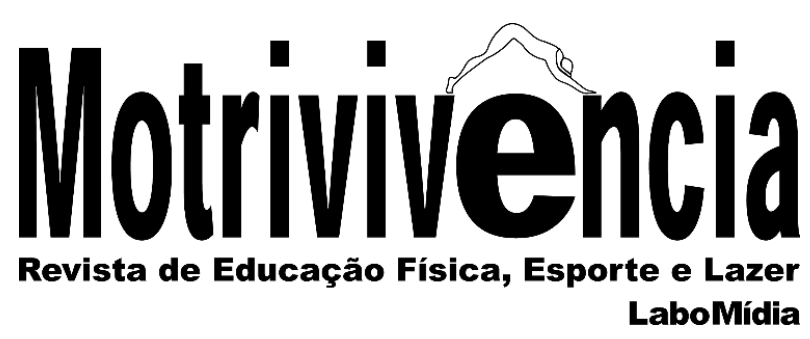

\title{
Produção de conhecimento e formação em psicologia do esporte no Amazonas
}

\section{RESUMO}

O presente estudo insere-se no âmbito da psicologia do esporte, especialidade e campo de atuação da psicologia. Considerando a interface entre as demais ciências do esporte, especialmente a educação física, buscou-se compreender a formação e a produção cientifica em psicologia do esporte no Amazonas. Mapearam-se possíveis produções, além de cursos credenciados de psicologia e educação física, suas respectivas estruturas curriculares visando identificar a inserção dessa área como disciplina, além de grupos e linhas de pesquisa. De modo geral os resultados apontam um contexto científico não consolidado com oferta de disciplinas de psicologia do esporte no bacharelado das formações em psicologia e educação física. Destaca-se o recente crescimento das monografias abordando a temática e a não divulgação dos resultados das pesquisas como uma problemática a ser enfrentada. Sugere-se a criação de alternativas no contexto acadêmico visando fomentar a divulgação das produções, assim como a relevante discussão sobre o campo da psicologia do esporte na formação acadêmica.

PALAVRAS-CHAVE: Psicologia do esporte; Educação física; Psicologia; Formação profissional; Produção científica
Matheus Vasconcelos Torres Especialista em Psicologia do Esporte Universidade Federal do Amazonas - UFAM Faculdade de Psicologia Manaus, Amazonas, Brasil matheusvasconcelos.psi@gmail.com https://orcid.org/0000-0001-5033-6141

Cristianne Almeida Carvalho

Doutora em Psicologia Social Universidade Federal do Maranhão - UFMA Departamento de Psicologia São Luís, Maranhão, Brasil cristianne.01@uol.com.br https://orcid.org/0000-0003-2793-7904

Ewerton Helder Bentes de Castro Doutor em Psicologia Universidade Federal do Amazonas - UFAM Faculdade de Psicologia Manaus, Amazonas, Brasil ewertonhelder@gmail.com https://orcid.org/0000-0003-2227-5278 


\title{
Knowledge production and professional qualification in sport psychology in the Amazonas
}

\begin{abstract}
This study is within the scope of sport psychology, specialty and professional field of psychology. Considering the interface between the other sports sciences, especially physical education, we sought to understand the qualification and scientific production in sport psychology in Amazonas. Possible productions were mapped and accredited courses in psychology and physical education, their respective curricular structures in order to identify the insertion of this area as a discipline, in addition to groups and lines of research. Generally, the results point to an unconsolidated scientific context with the offer of sports psychology courses in the Bachelor of Psychology and Physical Education courses. We highlight the recent growth of monographs addressing the theme and the non-disclosure of research results as a problem to be faced. It is suggested to create alternatives inserted in the academic context in order to promote the dissemination of productions, as well as the relevant discussion on the field of sport psychology in qualification.
\end{abstract}

KEYWORDS: Sports psychology; Physical education; Psychology; Professional qualification; Scientific production

\section{Producción de conocimiento y formación em psicologia del deporte en el Amazonas}

\section{RESUMEN}

Este estudio es parte del campo de la psicología del deporte, especialidad y campo de acción de la psicología. Considerando la interfaz entre las otras ciencias del deporte, especialmente la educación física, buscamos entender la formación y la producción científica en psicología del deporte en Amazonas. Fue mapeado las posibles producciones, los cursos autorizados de psicología y educación física, sus respectivas estructuras curriculares para identificar la inserción del campo como asignatura universitaria, además de grupos y líneas de investigación. En general, los resultados apuntan a un contexto científico no consolidado con la oferta de asignatura de psicología deportiva en los cursos de bachillerato en Psicología y Educación Física. Destacamos el reciente crecimiento de las monografías que abordan el tema y la falta de divulgación de los resultados de las investigaciones como un problema a enfrentar. Proponemos la creación de alternativas en el contexto académico para promover la difusión de producciones, así como la relevante discusión sobre el campo de la psicología del deporte en la formación académica.

PALABRAS-CLAVE: Psicología del deporte; Educación Física; psicología; Formación profesional; Producción científica 


\section{Introdução}

O presente artigo objetiva mapear o cenário de produções acadêmicas da psicologia do esporte (PE) no Amazonas a partir de um estudo descritivo, investigando as formações em psicologia e educação física, além das produções acadêmicas na região. A PE é uma das especialidades do saber psicológico reconhecida pela Resolução 013/2007 do Conselho Federal de Psicologia - CFP e caracteriza-se por um contexto de atuação direcionado às pessoas envolvidas em contextos esportivos e de exercício físico, dialogando com as demais ciências do esporte. Em decorrência dos frequentes avanços nos estudos, tem sido perceptível a expansão do seu campo de atuação e pesquisa no país.

No entanto, falar da presença da psicologia do esporte no Amazonas não é uma tarefa fácil, sendo necessário buscar um entendimento primordial para o avanço da área no estado. Para delinear uma trajetória sobre a área faz-se necessário situar brevemente o surgimento da psicologia como formação articulando as demais interfaces existentes com a educação física. Em seguida apresentase o método e os resultados encontrados.

\section{Contexto nacional da psicologia do esporte}

A psicologia do esporte no final do século XX era considerada uma área emergente no país e sua história coincide com os primórdios da psicologia geral no Brasil. Desde então a área tem se consolidado no país e a necessidade de reflexão sobre o seu contexto de atuação e acadêmico tem se evidenciado em diferentes regiões (VIEIRA, 2010; VIEIRA, JUNIOR e VIEIRA, 2013; ANDRADE et al., 2015). Porém, cabe lembra que sua trajetória histórica antecede a emancipação da psicologia enquanto profissão.

Rubio (1999) relembra que a PE no Brasil teve importantes contribuições a partir dos estudos de João Carvalhaes, profissional que detinha grande experiência com a psicometria e que atuou junto ao São Paulo Futebol Clube durante 20 anos a contar da década de 1950. Conforme Carvalho e Jacó-Vilela (2009), A participação de Carvalhaes representa um momento importante que favoreceu o reconhecimento da psicologia do esporte como campo de atuação da psicologia, porém a importância dos conhecimentos e fenômenos psicológicos junto ao desporto emergiu nos anos 1930, conforme pesquisa realizada nos primeiros periódicos da educação física (CARVALHO, 2019). 
Neste período destaca-se a Revista do Exército, onde médicos, filósofos e educadores discutem acerca de temáticas psicológicas, a exemplo de Plínio Olinto, professor do Instituto de Educação e Chefe do Serviço de Profilaxia do Hospital Nacional que publica o artigo "Educação física e educação psíquica" em 1933; Lourenço Filho, "Psicologia e educação física" em 1935; Airton Salgueiro de Freitas, “A Educação física sob o ponto de vista psicológico” em 1938. Observa-se que os fenômenos ou teorias psicológicas já eram abordados no universo da formação em educação física na primeira metade do século XX por profissionais de outras áreas. Estes apontamentos delineiam o surgimento de um esboço da psicologia do esporte no Brasil (CARVALHO e JACÓ-VILELA, 2009).

Porém, este contexto de surgimento da disciplina no país, atrelada à marcante presença da educação física nas escolas do exército, é marcado por um contexto guiado pela perspectiva técnico biológica (AZEVEDO e MALINA, 2004). Tal fato atina para as repercussões significativas que a presença da educação física nas escolas do exército teve em seu fazer pedagógico, mas por outro lado, em como "a psicologia é determinante na construção do pensamento pedagógico do Exército e na área da Educação Física em específico" (NETO, 1999, p. 60). Este contexto nos leva a intuir o aspecto basilar da presença dos conteúdos oriundos do saber psicológico na formação de profissionais de educação física.

Já em relação a busca da psicologia para se firmar como profissão no Brasil, a partir dos anos 1950 as frentes acadêmicas e profissionais existentes da psicologia no país se mobilizaram para regulamentação da profissão que se consolidou pela Lei 4.119 de 1962. Este fato foi impulsionado pela necessidade desta ciência sair do patamar de disciplina e avançar para a aplicação de seus conhecimentos em áreas como educação e recursos humanos, especialmente na criação e na sistematização de muitos testes psicológicos que buscavam quantificar e aferir inteligência, comportamento, personalidade e outros temas considerados da esfera da psicologia. Este é o caminho seguido pela psicologia do esporte, tendo em vista a importante participação de João Carvalhaes neste movimento (RUBIO, 2007).

Concomitantemente Carvalho (2016) compreende que o conhecimento oriundo da psicologia aplicada ao trabalho passa a ser utilizado no universo esportivo. Isto se faz através dos instrumentos psicométricos e outros fundamentos, integralmente oriundos da psicologia industrial. Consequentemente entende-se que a orientação profissional seria capaz de indicar o melhor trabalho para cada homem, visando o bem-estar individual e social, a orientação desportiva indicará o melhor desporto ao desportista (RUBIO, 2007).

As contribuições de João Carvalhaes através da psicometria são significativas na psicologia do esporte e repercute na atualidade. O uso de instrumentos para avaliação de estresse, ansiedade, 
humor e motivação, em sua maioria importados do contexto clínico e organizacional, tornam-se corriqueiros. Porém, ainda há um risco de uso generalizado destes instrumentos, uma vez que não contemplam em sua totalidade as especificidades e características do contexto esportivo. Logo, isto faz com que a falta de discussão, ausência de instrumentos psicológicos validados para o contexto esportivo brasileiro e o despreparo profissional para a construção de novos instrumentos sejam alvo de discussão na área (SILVA et al., 2014).

Deste contexto histórico para cá os estudos na área da PE tem se aperfeiçoado e tem sido exitoso na construção de um arcabouço teórico e metodológico específico para debruçar-se em diferentes fenômenos. Isso tem possibilitado novas compreensões e o redimensionamento dos olhares sobre os atletas. Um movimento que tem ganhado força desde os anos 1990 com estudos sobre interação social, dinâmica dos grupos esportivos, os perfis de liderança, as mulheres no esporte e questões de gênero, bem como o papel da psicologia frente a reabilitação do atleta lesionado e/ou hospitalizado, e de outros atores do contexto esportivo, como as equipes de arbitragem (RUBIO, 2007; SIMÕES, 2003; DEBIEN et al., 2014; MEDEIROS, 2016; SOUSA, 2016; LIMA e RUBIO, 2017).

Sendo assim, evidencia-se o crescente movimento da psicologia do esporte brasileira e sua convergência para a diversificação dos estudos que atendem arbitragem, mídia, políticas públicas, gênero e outras vertentes que se incluem no campo esportivo, além da preparação psicológica do atleta de alto rendimento. Tais desdobramentos caracterizam o cenário nacional e apontam caminhos para o fortalecimento do campo profissional, porém, ainda se faz necessário atentar para as regionalidades presentes no país.

\section{Cenário de produções acadêmicas no amazonas}

Sobre as publicações científicas do país, cabe ressaltar que Vieira, Junior e Vieira (2013) apontam que a maioria das produções são oriundas dos programas de pós-graduação das universidades públicas, o que para o Amazonas, na área esportiva, torna-se uma problemática, tendo em vista que o primeiro curso de graduação em educação física inicia suas atividades em 1970, e sua primeira turma de pós-graduação é acolhida pelo Programa de Saúde, Sociedade e Endemias na Amazônia da Universidade Federal do Amazonas que teve sua abertura apenas no ano 2017.

Soma-se a esse fato a jovialidade da psicologia no Amazonas, uma vez que seus primeiros cursos têm início nos anos de 1992 e 1995, respectivamente no Centro Universitário Luterano e na Universidade Federal do Amazonas, em acordo com dados do e-MEC. Concomitantemente, nesta 
última, está em atividade o único programa de pós-graduação em psicologia no Amazonas com abertura da primeira turma em 2012.

Sobre este quadro, Andrade et al. (2015) demonstram que os estudos em psicologia do esporte no país estão concentrados na região sul e sudeste. Já Vilarino et al. (2017) aponta o recente crescimento da produção na região nordeste, porém, ainda é evidente o distanciamento da região norte do Brasil neste cenário, o que ressalta a necessidade de produções que impulsionem a PE no Amazonas.

Dessa forma, levantou-se questões referentes à formação e produção científica no Amazonas dentro do campo temática: Quantos cursos tem ofertado disciplinas relacionadas à psicologia do esporte? Quantos grupos de pesquisa estão em atividade em cada área e quais apresentam linhas de pesquisa voltados à $\mathrm{PE}$ ? O que há de produção científica relacionada com a temática no Amazonas? Em que momento ocorreram estas produções? Quais são os temas mais recorrentes?

\section{Métodos}

Para responder as questões levantadas por este estudo, os procedimentos metodológicos adotados foram embasados na metodologia de estudo do estado da arte, tendo como foco o conhecimento em psicologia do esporte no Amazonas. Compreende-se estado da arte enquanto tentativa de conhecer um contexto de produção científica ainda desconhecido com o intuito de delinear caminhos para futuras pesquisas que preencham as lacunas existentes do conhecimento (VARGAS, HIGUITA e MUÑOZ, 2015).

Para Vargas, Higuita e Muñoz (2015), a metodologia do estado da arte além de proporcionar a descoberta de um contexto de produção desconhecido, possibilita a construção de reflexões, críticas, reconhecimento e construção do campo de estudo. Concomitantemente, sua metodologia propõe as seguintes etapas que foram seguidas neste estudo: Passo 1 - Planejamento: Consistiu em elaborar as perguntas e identificar as principais bases de dados a serem encontradas as informações buscadas; Passo 2 - Desenho: Realizou-se a síntese de dados; Passo 3 - Análise e formalização: Foi realizada a análise dos dados encontrados (VARGAS, HIGUITA e MUÑOZ, 2015).

Logo, visando ter um panorama geral do campo acadêmico da psicologia do esporte no Amazonas, buscaram-se os cursos de psicologia e educação física oferecidos no Amazonas através da base de dados oficial do Ministério da Educação, o e-MEC, onde constam as Instituições e Cursos de Educação Superior ativos. Para consulta foram considerados os cursos de psicologia e educação física localizados no Amazonas que estivessem em atividade e fossem ofertados na 
modalidade presencial. Foram excluídos os cursos em processo de extinção. Posteriormente foi analisada a presença da disciplina de psicologia do esporte nas estruturas curriculares ou similares dos cursos. As matrizes de ambos os cursos foram consultadas através do acesso ao portal institucional. Para identificar os grupos de pesquisa no Amazonas, foi realizada busca no Diretório dos Grupos de Pesquisa no Brasil com o termo "Psicologia do esporte" considerando como região “Amazonas". Consequentemente foram analisadas as linhas de pesquisa dos respectivos grupos para identificar a presença do campo de estudo.

Complementando os dados e atentando para as produções na área foi realizado levantamento bibliográfico através de buscas no Sistema de Bibliotecas da Universidade Federal do Amazonas UFAM a fim de identificar produções na área, assim como análise de 92 currículos Lattes de pesquisadores sugeridos pelo site do Conselho Nacional de Desenvolvimento Científico e Tecnológico - CNPq com o intuito de identificar monografias, artigos e publicações em anais de congressos. Para consulta foram escolhidos os termos "Psicologia do esporte" e "Amazonas", tendo em vista o fornecimento das informações pela Plataforma Lattes ao filtrar região e palavras-chaves através da busca por assunto. Para estes dados foi definido recorte temporal de 2003-2016.

Tabela 1: Procedimentos para coleta de dados

\begin{tabular}{|c|c|c|}
\hline Cursos em Atividade & Grupos de Pesq & ões Científicas \\
\hline $\begin{array}{l}\text { 1. Consulta ao e-MEC; } \\
\text { 2. Verificação de atividade do } \\
\text { curso; } \\
\text { 3. Consulta às grades } \\
\text { curriculares; } \\
\text { 4. Identificação das } \\
\text { disciplinas. }\end{array}$ & $\begin{array}{l}\text { 1. Consulta ao Diretório dos } \\
\text { Grupos de Pesquisa no } \\
\text { Brasil; } \\
\text { 2. Busca por termo "Psicologia } \\
\text { do Esporte" no } \\
\text { "Amazonas"; } \\
\text { 3. Identificação dos Grupos e } \\
\text { linhas de pesquisa. }\end{array}$ & $\begin{array}{l}\text { 1. Fontes: Plataformas } \\
\text { Lattes e Sistema de } \\
\text { Bibliotecas da UFAM; } \\
\text { 2. Busca pelo termo } \\
\text { "Psicologia do Esporte" } \\
\text { e "Amazonas"; } \\
\text { 3. Identificação das } \\
\text { produções. }\end{array}$ \\
\hline
\end{tabular}

Fonte: Elaborado pelos autores.

Em consulta prévia, as buscas em periódicos locais e outras bases de dados foram descartadas por não retornarem dados através da utilização dos descritores, assim como as bibliotecas universitárias que em visita prévia de campo demonstraram carecer de organização destinada às monografias produzidas durante a graduação, o que viria a dificultar ou enviesar as informações coletadas nas diferentes instituições.

Ao final foram considerados apenas os cursos regularizados, os grupos de pesquisa certificados, currículos indicados pela plataforma lattes atualizados nos últimos quatro anos e trabalhos que resultaram em produções, tais como artigos em periódicos, capítulos de livro, resumos 
e trabalhos completos em anais de eventos, monografias e dissertações. A coleta e análise dos dados foi realizada durante os anos de 2017 e 2018.

\section{Resultados}

O crescimento de um campo de atuação em uma profissão, como o caso da psicologia do sporte, está relacionado com a forma que tem sido apresentado em sua formação profissional. Vieira, Junior e Vieira (2013) destacam que os estudos sobre PE estão concentrados nos programas de pós-graduação em educação física, o que ainda reflete uma aparente dissociação da área dentro dos grupos de pesquisa da psicologia.

No levantamento realizado através do e-MEC e na busca por informações nas universidades com cursos de educação física e psicologia regularizados e oferecidos em modalidade presencial, constatou-se a existência de 61 cursos de educação física, sendo 34 licenciaturas e 27 bacharelados e dez cursos de psicologia na modalidade de bacharelado, conforme pode ser verificado na figura 1 :

Figura 1: Cursos de psicologia e educação física em atividade no Amazonas.

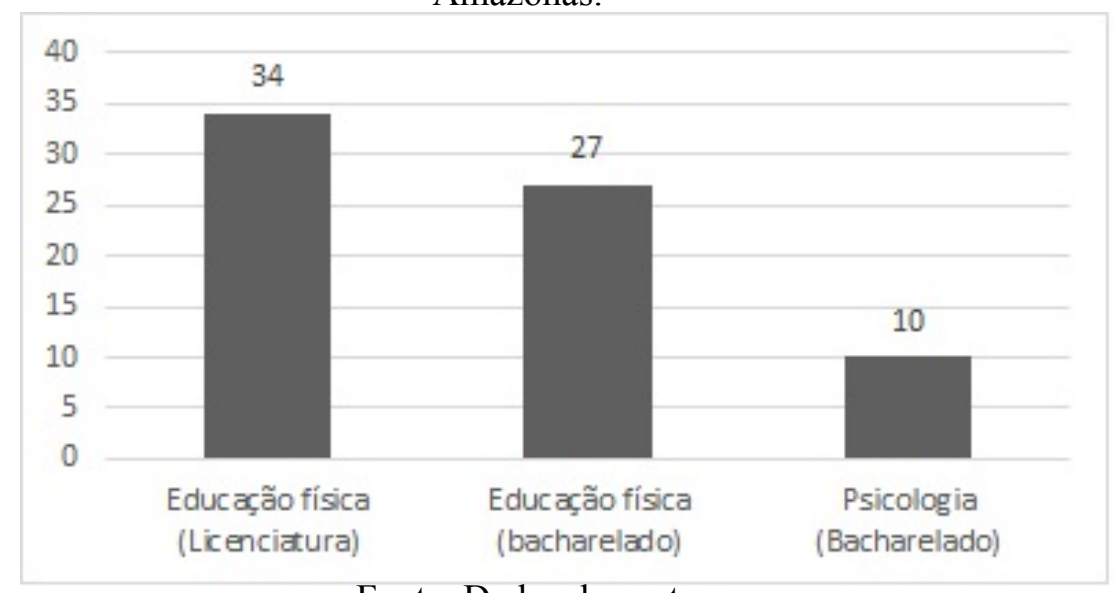

Fonte: Dados dos autores.

Ainda sobre estes dados, cabe ressaltar que foi possível identificar que os cursos de psicologia estão concentrados na capital amazonense, ofertando 2.246 vagas. Já os cursos de educação física, que ofertam 6.400 vagas, destacam-se pelo processo de interiorização através das universidades públicas de âmbito estadual e federal. Tal informação torna-se relevante considerando as características territoriais do Amazonas marcadas pelas singularidades da Amazônia e da dificuldade de acesso ao ensino superior na região.

Em relação a oferta da disciplina de psicologia do esporte, verifica-se a presença na totalidade dos cursos de bacharelado em educação física em caráter obrigatório, enquanto nos 
cursos de licenciatura são ofertadas apenas disciplinas de psicologia da educação e psicologia do desenvolvimento. Nos cursos de bacharelado em psicologia a disciplina apresenta-se com menor frequência. A figura 2 ilustra tal contexto:

Figura 2: Caráter de oferta da disciplina de psicologia do esporte nos cursos de educação física e psicologia.

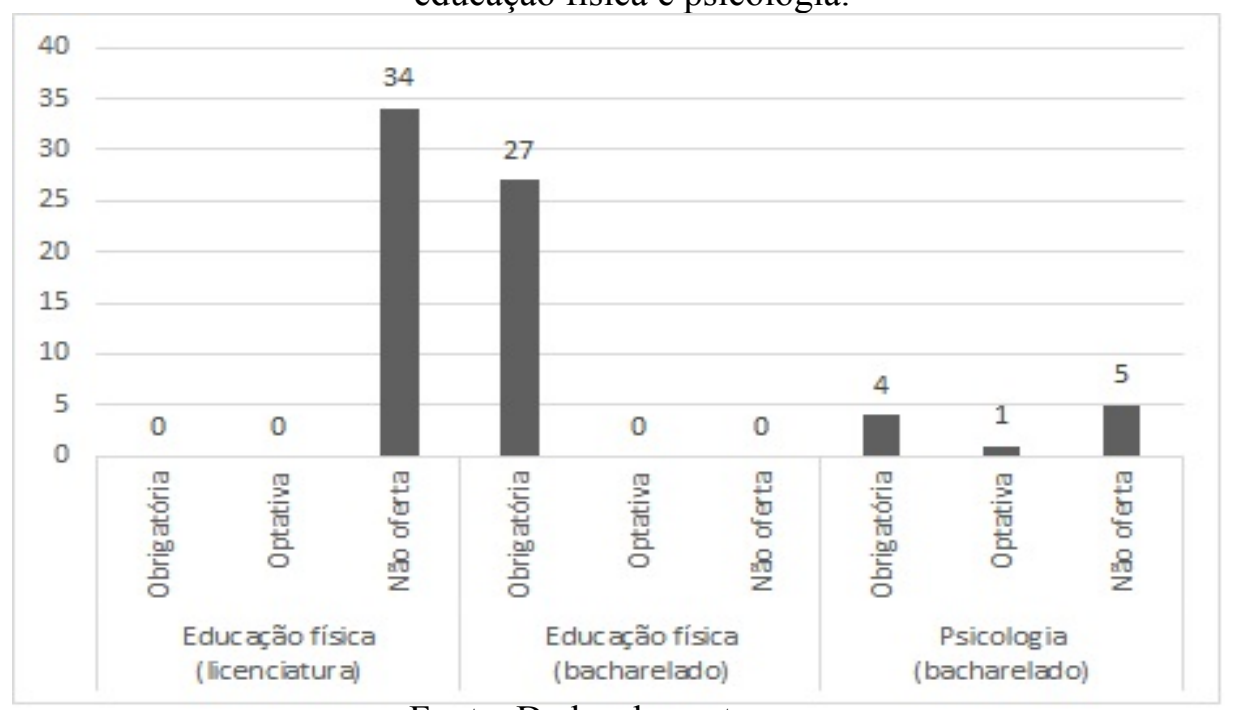

Fonte: Dados dos autores.

Em outro conjunto de dados levantados e apresentados na figura 3, foi verificado junto ao Diretório de Grupos de Pesquisa no Brasil, os grupos em atividade relacionados aos cursos deste estudo. Conforme a tabela a seguir foi possível verificar o quantitativo de grupos em atividade indicados pela consulta:

Figura 3: Grupos de pesquisa em atividade na área da psicologia e da educação física no Amazonas.

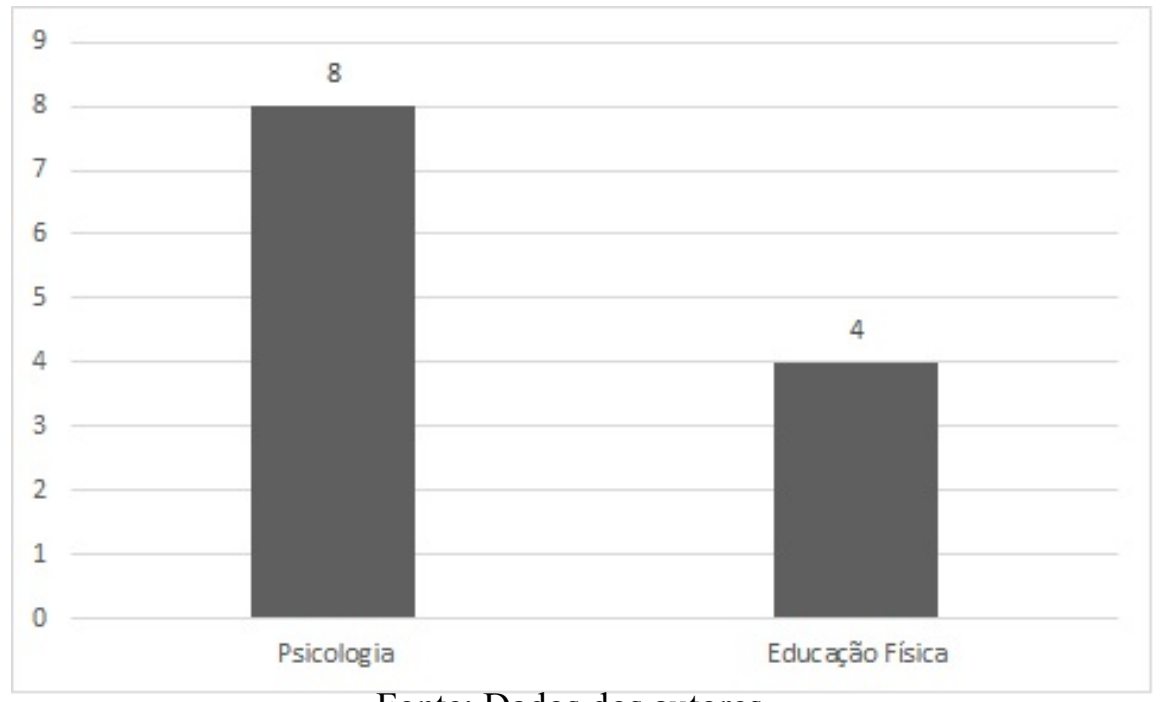

Fonte: Dados dos autores. 
A maioria dos grupos indicados pela consulta concentram-se na área da psicologia, todavia, apenas o Grupo de Pesquisa de Psicologia Cognitiva: Criatividade e Corporeidade têm entre suas linhas de pesquisa a psicologia do esporte. Este atualmente encontra-se lotado dentro da Faculdade de Educação Física e Fisioterapia da Universidade Federal do Amazonas - FEFF/UFAM.

Diante desse cenário, as informações sobre a produção acadêmica através da análise 92 currículos indicados pela plataforma lattes e da consulta ao Sistema de Bibliotecas da Universidade Federal do Amazonas, nos possibilitou identificar o seguinte quantitativo de registros de produções nos currículos conforme a figura 4 :

Figura 4: Produções em psicologia do esporte no Amazonas registradas em currículos na plataforma lattes.

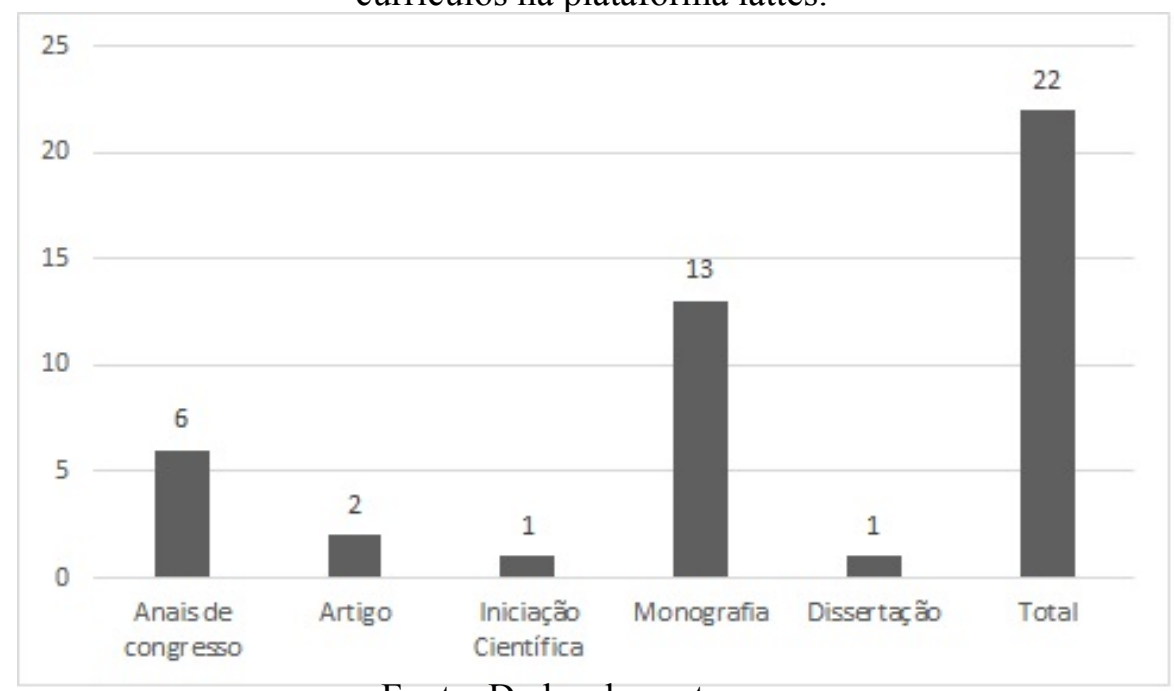

Fonte: Dados dos autores.

Dessa forma, foram encontrados registros de artigos científicos, publicações em anais de eventos e monografias concentrados em 9 currículos, além de uma dissertação intitulada "Redescobrindo ser-si-mesmo: a existencialidade de mulheres praticantes de pole dance" oriundo do programa de pós-graduação em psicologia da UFAM. Enfim, destacam-se pela veiculação e possibilidade de acesso aos materiais os trabalhos de Siqueira e Vieira (2013) sobre o abandono precoce de mulheres na natação, Ferreira (2015) sobre a vivência de mulheres praticantes de pole dance, além de Torres e Castro (2017) em monografia concluída em 2016 sobre a prática de futsal por mulheres. Não foi possível identificar publicização das demais produções.

A partir desta análise constatou-se maior interesse pela pesquisa na área de psicologia do esporte ainda na graduação, ressaltado pela quantidade de monografias $(n=13)$ registradas na plataforma. Registra-se que a maioria dos trabalhos encontrados se concentram nos cursos de educação física $(n=18)$ e, apenas duas monografias, um trabalho publicado em anais de congresso e uma dissertação foram produzidas nos cursos de psicologia. 
Além disso, observa-se que há constante variação nas produções relacionadas à área, possivelmente indicando interesses isolados pela temática. Destaca-se que durante o período de 2009 a 2016 concentram-se a maior parte das produções $(n=15)$. Na figura 5 a seguir é possível vislumbrar a apresentação destes dados entre os períodos de 2003-2016:

Figura 5: Variação da produção de trabalhos durante o período de 2003-2016 no Amazonas.

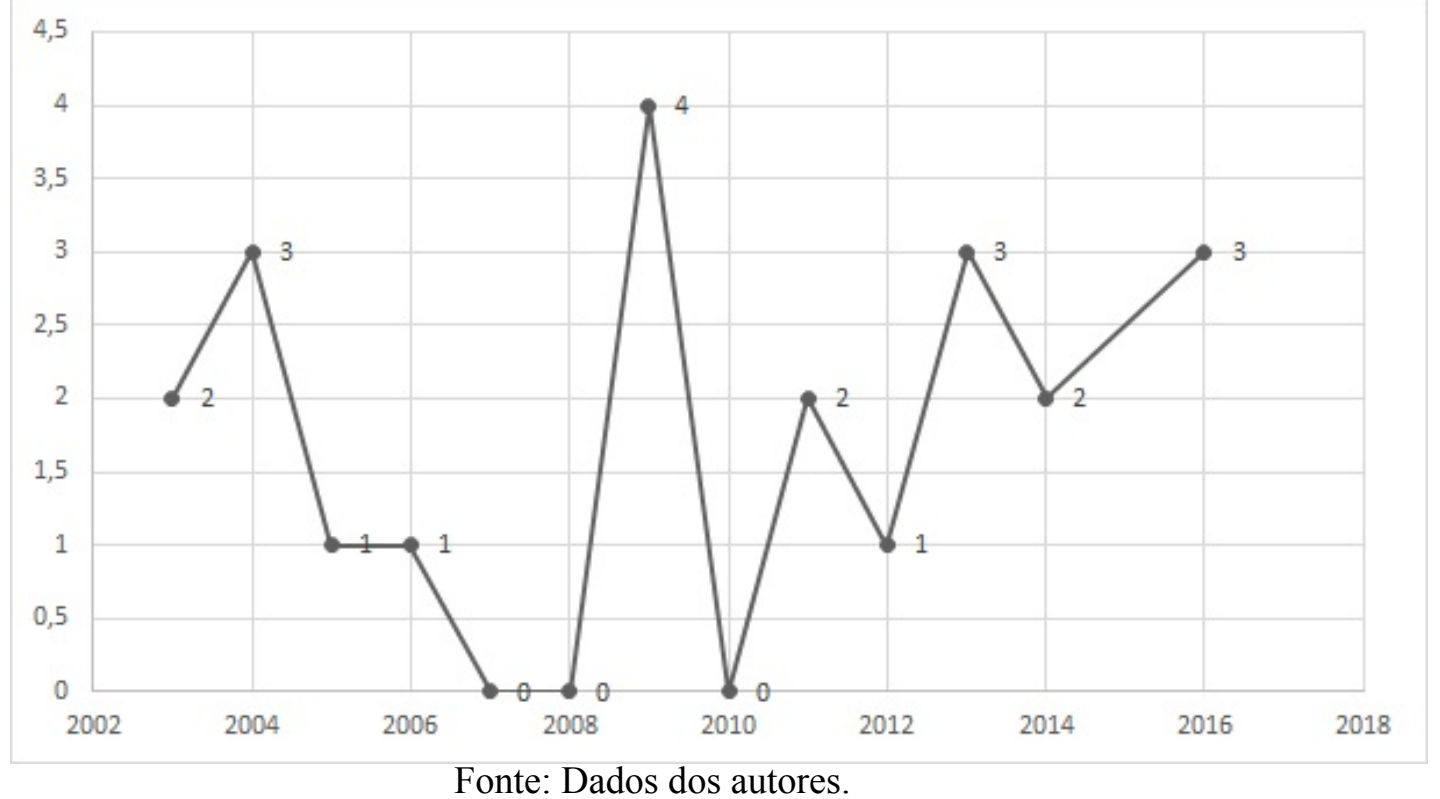

Quanto as temáticas desenvolvidas ao longo deste período apresentadas na figura 6, identificou-se uma pluralidade de temas (figura 6), tendo destaque os trabalhos sobre motivação $(n=6)$ e iniciação esportiva $(n=3)$. Outro grupo de publicações está relacionado aos estudos sobre estresse $(n=2)$ e ressocialização $(n=2)$. No último grupo aparecem atenção, estados de humor, lesões, estilo de vida, memória, intervenção psicológica no esporte, inteligências múltiplas, imagem corporal e identidade, com um estudo cada. 
Figura 6: Temáticas das produções registradas na Plataforma Lattes entre os anos de 2003 e 2016.

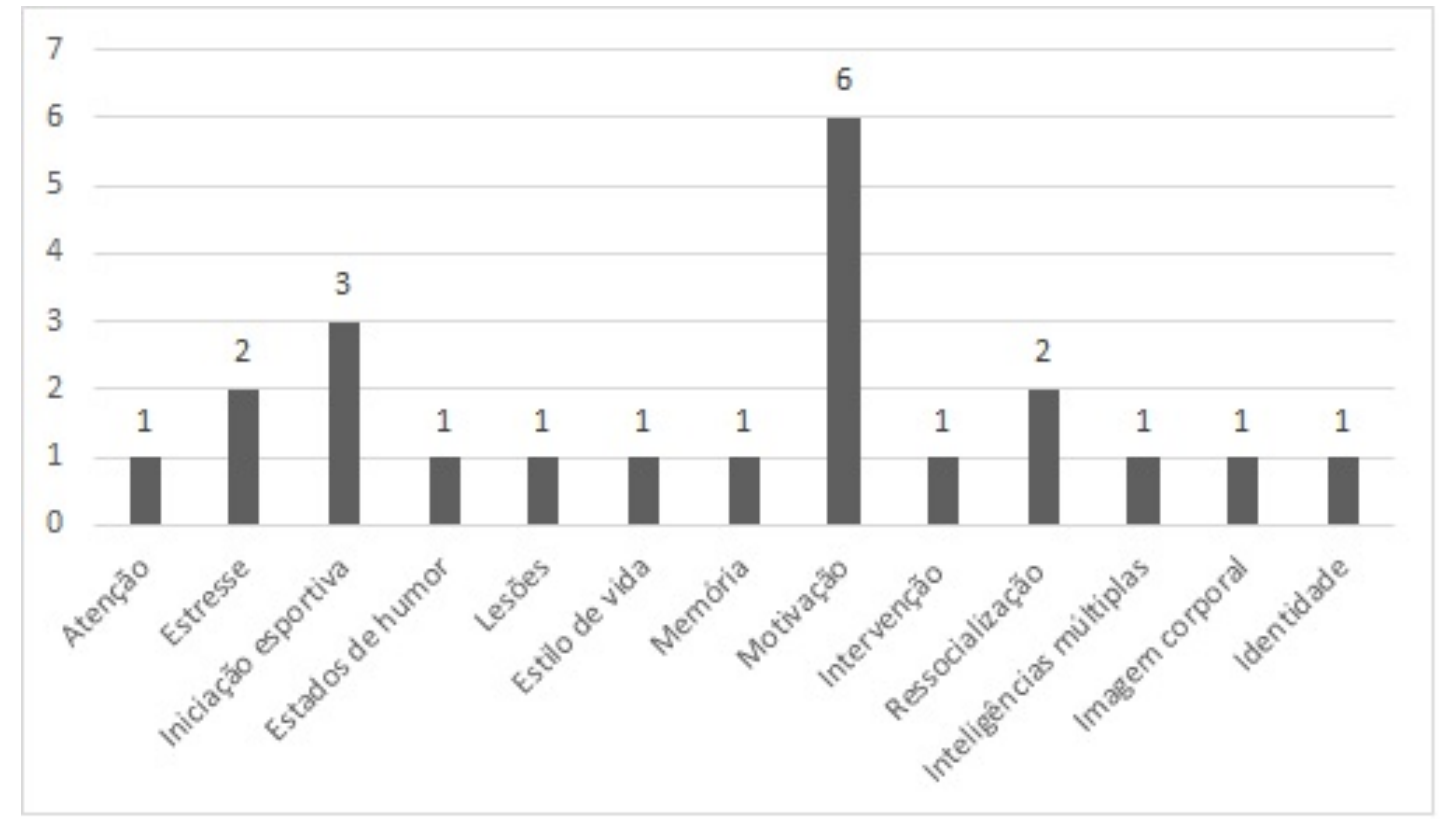

Fonte: Dados dos autores.

Por fim, com base nos dados coletados para análise neste estudo, foi possível organizar um conjunto de informações que representam o contexto pesquisado. Tais informações ganham destaque quanto discutidos em articulação com a literatura já existente sobre o campo acadêmico da psicologia do esporte. Consequentemente, faz-se necessário lançar luz aos resultados.

\section{Discussão}

Os resultados do estudo apontam possibilidades para compreensão do panorama sobre a psicologia do esporte no Amazonas. Através do conjunto de informações encontradas através da metodologia de busca proposta por este estudo, faz-se necessário tecer discussões sobre o contexto encontrado.

Sobre os dados relacionados ao contexto de formação, Vieira et al. (2010) apontam que uma possível explicação para este contexto de não favorecimento está relacionada à contratação de profissionais que não são psicólogos do esporte para atuar junto às equipes de esportes individuais e/ou coletivos, e até mesmo em projetos sociais. Esta problemática poderia associar-se com a ausência da disciplina de psicologia do esporte nas grades curriculares dos cursos de psicologia. Porém, apesar dos resultados apresentarem a presença da disciplina nos cursos, seria necessário aprofundamento na conjuntura formativa das instituições que garantem a oferta da disciplina, o que 
demandaria novos estudos que buscassem a compreensão do compromisso com este campo aplicado no projeto político pedagógico dos cursos.

Concomitantemente, a especialização neste campo configura-se como um desafio para as dimensões e especificidades do território brasileiro, especialmente na região norte. A ausência de cursos de pós-graduação presenciais na área na região norte, relega as possibilidades de formação de profissionais à lógica de mercado dos cursos de especialização a distância. Isto reflete diretamente na possibilidade de um processo formativo de qualidade. Lopes et al. (2015) destaca que o isolamento geográfico do Norte contribui para o quadro econômico, político e social de seus estados. Não descartaríamos que este isolamento reflete no desenvolvimento da ciência na região, neste caso em especial, no campo da psicologia do esporte e no contexto esportivo como um todo.

A situação supracitada é confirmada pela Capes (2016a) em seu documento de avaliação sobre a psicologia no país. Uma vez que sobre os cursos de programa de pós-graduação (PPG) é apontado que dos 74 programas, apenas 5,9\% $(n=5)$ estão na região norte do país, sendo que dos sete cursos presentes nestes programas, apenas o curso de mestrado em psicologia da Universidade Federal do Amazonas representa a região. No Brasil, os programas de pós-graduação têm apresentado importância para o avanço na produção científica e sua repercussão em âmbito nacional e internacional. (BASTOS, 2010). A existência de programas ativos com linhas de produção no campo da psicologia do esporte seria relevante para promover impactos na região.

Tal afirmação é constatada ao verificar que até o ano de 2012, no Brasil, havia 14 PPGs educação física e psicologia com linhas de pesquisa dedicadas à psicologia do esporte, sendo estes concentrados em "instituições - majoritariamente públicas - nas regiões Sul e Sudeste do país." (VIEIRA, JUNIOR e VIEIRA, 2013, p. 502). Tal cenário é marcador das iniquidades regionais presentes, porém nos traz à tona questionamentos sobre os desafios interpostos ao norte do país, bem como as estratégias necessárias para contornar tal cenário.

Além disso, voltando-se para o contexto da produção acadêmica, é válido lembrar da importância da multidisciplinaridade na área esportiva destacada por Gaertner (2007), uma vez que a aproximação entre a psicologia e a educação física, assim como as demais ciências do esporte se faz fundamental e esta é uma possível estratégia a ser adotada visando o crescimento da área.

Ressalta-se que no cenário amazonense, apesar da ausência de um programa de pósgraduação em educação física como aponta o documento de avaliação da Capes (2016b), tem havido interesse por estudantes e pesquisadores da região sobre os assuntos pertinentes à psicologia do esporte, conforme os dados apresentados sobre a variação da produção entre os anos 2003-2016. Diferente do estudo de Vieira, Júnior e Vieira (2013), os estudos encontrados indicam uma produção concentrada na graduação. 
Tendo em vista este fato, destaca-se como aspecto positivo a concentração de estudos durante a graduação através das monografias $(n=13)$ registradas na plataforma lattes. Este resultado trará uma importante comparação com dados futuros encontrados em posterior análise. Ainda ressalta-se que a estes dados associamos a exigência de conhecimentos em psicologia na graduação em educação física, tendo em vista a presença obrigatória em todas as estruturas curriculares analisadas nos cursos do Amazonas, onde encontramos as seguintes disciplinas: Psicologia do desenvolvimento e psicologia da educação para a licenciatura e psicologia do esporte para o bacharelado da área.

O fato deste estudo ser inédito também exigirá futuros levantamentos para acompanhar o desenvolvimento da área. O estudo de Andrade (2015) é um contributo ao estudo de Vieira, Junior e Vieira (2013) e conseguem apontar um crescimento nos estudos sobre psicologia do esporte que se justificou pelo crescimento dos PPG em psicologia. Logo, os dados aqui apresentados também corroboram com os estudos supracitados que indicam a concentração dos estudos da psicologia do esporte nas regiões sul e sudeste, seguidas pelas regiões centro-oeste e nordeste, em decorrência dos avanços dos programas de pós-graduação.

O contexto emergente da psicologia do esporte na região norte do país nos indica justificativas para os dados encontrados. Considerando as informações coletadas, verifica-se um distanciamento da psicologia em relação ao contexto esportivo regional, o que torna evidente que o Amazonas tem o desafio de intensificar estudos e pesquisas, além do incentivo à atuação na área.

A impossibilidade de consultar todos os trabalhos minunciosamente, seja pelo registro realizado unicamente na plataforma lattes ou pela ausência de um controle de registros efetivo na produção das monografias, nos levou a evitar uma análise aprofundada destes trabalhos. Tal fato poderia contribuir de outras maneiras com os estudos de Salvador et al. (2007), Vieira, Junior e Vieira (2013) e Andrade (2015) que apontam tendências para estudos de revisão e descrição na Psicologia do Esporte. Porém, a concentração de estudos sobre motivação, estresse, estados de humor e iniciação esportiva são similaridades encontradas com os dados dos autores supracitados.

\section{Considerações finais}

Investigar o campo acadêmico de uma área através de aspectos relevantes presentes na formação, assim como de suas produções, possibilita preencher uma lacuna existente, principalmente quando falamos de uma região que necessita de profissionais que consolidem seu contexto de atuação e pesquisa. 
Os resultados encontrados neste levantamento tornam evidente que estudos mais aprofundados e sistematizados são necessários para compreender a fundo o contexto local, bem como é explícita a necessidade de fomento à pesquisa sobre a temática nos PPG's da região. Iniciativas de diálogo entre profissionais de Psicologia e Educação Física possivelmente serão um caminho necessário para alavancar a criação de campos de atuação, e quiçá pesquisas na área. Ao mesmo tempo explana-se a importância de suscitar reflexões sobre o esporte no Amazonas, bem como a formação voltada para este campo, cogitando a possibilidade e porventura a necessidade de incentivar a discussão ao longo da graduação, assim como na pós-graduação.

Concomitantemente, a criação de periódicos, a organização de eventos e até a aproximação com as entidades representativas dos profissionais da área no país, poderão fortalecer um possível movimento em direção ao crescimento na área, além de servir como base para futuras consultas e abrir caminhos para solidificação da área no norte do país. Estes resultados revelam não somente a necessidade de nós, profissionais em atuação, pesquisadores e estudantes no Amazonas de zelar pela área esportiva, assim como das demais regiões em contribuir com nossas possíveis iniciativas.

Além disso, é importante destacarmos que este estudo lidou com limitações. A primeira delas diz respeito a análise dos currículos indicados pela plataforma lattes, uma vez que apenas oito currículos foram selecionados por possuírem registros de trabalhos oriundos das problemáticas da região. Concomitantemente, alguns trabalhos foram passíveis de identificação somente através do registro do orientador ou de membro da banca das monografias, apontando possível carência de orientações sobre o uso do sistema à estudantes, o que nos indica ausência de estratégias para promover publicações nesta fase da formação. Tal fato necessita de reflexões aprofundadas, uma vez que a veiculação dos trabalhos de pesquisa realizados em âmbito universitário deve ser tratada como compromisso ético-político pelas instituições e poderia ter possibilitado a leitura dos conteúdos na íntegra.

O fortalecimento da profissão em torno da psicologia do esporte pode enfim contribuir para emancipação de um novo campo profissional para a psicologia no Amazonas e possivelmente gerar resultados relevantes ao contexto de estudos no Brasil, bem como, sem relegar à segundo plano, contribuir com o cuidado aos atletas e à população local que tem buscado debruçar-se no cotidiano das atividades físicas e esportivas.

\section{REFERÊNCIAS}

ANDRADE, Alexandre et al. Psicologia do esporte no Brasil: revisão em periódicos da psicologia. Psicologia em Estudo, v. 20, n.2, 2015. 
AZEVEDO, Ângela Celeste Barreto de; MALINA, André. Memória do currículo de formação profissional em educação física no Brasil. Revista Brasileira de Ciências do Esporte, v. 25, n. 2, 2004.

CAPES. Coordenação de Aperfeiçoamento de Pessoal de Nível Superior. Documento de Avaliação da Área de Psicologia. Brasil, Governo Federal: Coordenação de Antonio Virgílio Bittencourt Bastos, 2016a.

CAPES. Coordenação de Aperfeiçoamento de Pessoal de Nível Superior. Documento de Avaliação da Área de Educação Física. Brasil, Governo Federal: Coordenação de André Luiz Felix Rodcki, 2016b.

CARVALHO, Cristianne Almeida. JACÓ-VILELA, Ana Maria. Psicologia do esporte no Brasil em dois tempos: Uma história contada e uma história a ser contada. Anais do XV Encontro Nacional da ABRAPSO, p. 1-7, 2009. Disponível em

http://abrapso.org.br/siteprincipal/index.php?option=com content\&task=blogsection\&id=18\&Itemi d=92. Acesso em: 12 nov. 2018.

CARVALHO, Cristianne Almeida. Psicologia do Esporte: Construindo sua história a partir da educação física: Revista Brasileira de Psicologia do Esporte, v.8, n.1, 2016.

CARVALHO, Cristianne Almeida. A psicologia como ciência do esporte e do exercício no Brasil. In Erick Conde et al. (Orgs.) Psicologia do Esporte e do Exercício: Modelos teóricos, pesquisa e intervenção. 1. ed. - São Paulo: Pasavento, 2019.

DEBIEN, Paula Barreiros et al. O estresse na arbitragem de ginástica rítmica: uma revisão sistemática. Journal of Physical Education, vol. 25, n3, 2014.

FERREIRA, Carolina Fernandes. Re-Descobrindo Ser-Si-Mesmo. A existencialidade de mulher praticantes de pole dance. Dissertação (Mestrado em psicologia), 80 folhas. Programa de PósGraduação em Psicologia, Universidade Federal do Amazonas - UFAM, Manaus, 2015.

GAERTNER, Gilberto. Psicologia e Ciências do Esporte. Curitiba, Brasil: Juruá, 2007.

LIMA, Luciana Tabarini. RUBIO, Katia. O atleta e a experiência de hospitalização. Revista Brasileira de Psicologia do Esporte, v.6, n.3, 2017.

LOPES, Jaíza Gomes Duarte, et al. Pobreza multidimensional: Uma aplicação à região norte do Brasil. Dissertação de Mestrado. Pontifícia Universidade Católica do Rio Grande do Sul, 2015.

MEDEIROS, Clarice. Lesão e dor no atleta de alto rendimento: O desafio do trabalho da psicologia do esporte. Psicologia Revista, v.25, n.2, 2016.

NETO, Amarílio Ferreira. A pedagogia no exército e na escola: A Educação Física (1920-1945). Motrivivência, n. 13, p. 35-62, 1999.

RUBIO, Kátia. A psicologia do esporte. Histórico e áreas de atuação e pesquisa. Psicologia:

Ciência e Profissão. v.19, n.3, 1999. 
RUBIO, Kátia. Da psicologia do esporte que temos à psicologia do esporte que queremos. Revista Brasileira de Psicologia do Esporte, v.1, n.1, 2007.

SALVADOR, Simone. et al. Análise da produção científica em psicologia do esporte. Revista Iberoamericana de psicologia del ejercicio y el deporte, v.2, n.1, 2007.

SILVA, Andressa Melina Becker da. et al. Instrumentos aplicados em estudos brasileiros de psicologia do esporte. Estudos interdisciplinares em psicologia, v.5, n.2, 2014.

SIMÕES, Antonio Carlos. Mulher \& esporte. Editora Manole Ltda, 2002.

SIQUEIRA, Thomas Décio Abdalla. VIEIRA, Raquel Siqueira de Carvalho. Desmotivation and leavin early. A study in a population of adolescent female swimming in Manaus. Revista de psicologia da IMED, v.5, n.2, p.121-125, 2013.

SOUSA, Marta Aparecida Magalhães. Um olhar para os árbitros de futebol. Revista Brasileira de Psicologia do Esporte, v. 6, n. 1, 2016.

TORRES, Matheus Vasconcelos. CASTRO. Ewerton Helder Bentes de. Ser-mulher-praticante de futsal: compreendendo o mundo vivido sob a ótica da fenomenologia. In: Ewerton Helder Bentes de Castro (org.) Fenomenologia e Psicologia: As teorias e práticas de pesquisa. 1.ed. Curitiba: Appris, v.1, p. 139-150, 2017.

TOURINHO, Emannuel Zagury. BASTOS, Antonio Virgilio Bittencort. Desafios da pós-graduação em psicologia no Brasil. Psicologia: Reflexão e Crítica, v.23, n.1, 2010.

VARGAS, Maricelly Gómez; HIGUITA, Catalina Galeano; MUÑOZ, Dumar Andrey Jaramillo. El estado del arte: una metodología de investigación. Revista Colombiana de Ciencias Sociales, v. 6, n. 2, p. 423, 2015.

VIEIRA, Lenamar Fiorese. et al. Psicologia do esporte: Uma área emergente da psicologia.

Psicologia em estudo, v.15, n.2, 2010.

VIEIRA, Lenamar Fiorese. JUNIOR, José Roberto Andrade do Nascimento. VIEIRA, José Luiz Lopes. O estado da arte da pesquisa em psicologia do esporte no Brasil. Revista de Psicología del deporte, v.22, n.2, 2013.

VILARINO, Guilherme Torres. et al. Análise dos grupos de pesquisa em psicologia do esporte e do exercício no Brasil. Revista Brasileira de Ciências do Esporte, v.39, n.4, 371-379, 2017.

\section{NOTAS DE AUTOR}

\section{AGRADECIMENTOS}

Agradeço as orientações do professor Dr. Ewerton Helder Bentes de Castro e da professora Dra. Cristianne Almeida Carvalho e todos os momentos que se fizeram presentes, assim como a dedicação incessante de todos aqueles que atuam em defesa da universidade pública. 


\section{CONTRIBUIÇÃO DE AUTORIA}

Concepção do manuscrito: M. V. Torres, C. A. Carvalho, W. H. B. Castro.

Coleta de dados: $M$. V. Torres.

Análise de dados: M. V. Torres, C. A. Carvalho.

Discussão dos resultados: $M$. V. Torres, C. A. Carvalho.

Produção do texto: M. V. Torres, C. A. Carvalho, W. H. B. Castro.

Revisão e aprovação: C. A. Carvalho, W. H. B. Castro.

\section{FINANCIAMENTO}

Não se aplica.

\section{CONSENTIMENTO DE USO DE IMAGEM}

Não se aplica.

\section{APROVAÇÃO DE COMITÊ DE ÉTICA EM PESQUISA}

Não se aplica.

\section{CONFLITO DE INTERESSES}

Não se aplica.

\section{LICENÇA DE USO}

Os autores cedem à Motrivivência - ISSN 2175-8042 os direitos exclusivos de primeira publicação, com o trabalho simultaneamente licenciado sob a Licença Creative Commons Attribution Non-Comercial ShareAlike (CC BY-NC SA) 4.0 International. Esta licença permite que terceiros remixem, adaptem e criem a partir do trabalho publicado, desde que para fins não comerciais, atribuindo o devido crédito de autoria e publicação inicial neste periódico desde que adotem a mesma licença, compartilhar igual. Os autores têm autorização para assumir contratos adicionais separadamente, para distribuição não exclusiva da versão do trabalho publicada neste periódico (ex.: publicar em repositório institucional, em site pessoal, publicar uma tradução, ou como capítulo de livro), com reconhecimento de autoria e publicação inicial neste periódico, desde que para fins não comerciais e compartilhar com a mesma licença.

\section{PUBLISHER}

Universidade Federal de Santa Catarina. Programa de Pós-Graduação em Educação Física. LaboMídia - Laboratório e Observatório da Mídia Esportiva. Publicado no Portal de Periódicos UFSC. As ideias expressadas neste artigo são de responsabilidade de seus autores, não

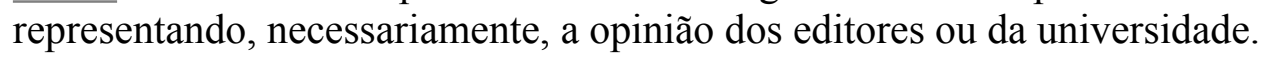

\section{EDITORES}

Mauricio Roberto da Silva, Giovani De Lorenzi Pires, Rogério Santos Pereira.

\section{HISTÓRICO}

Recebido em: 12 de Novembro de 2018.

Aprovado em: 22 de Julho de 2019. 